Methods 109 EU sites dosed 1006 patients. Safety data were recorded at routine clinic visits for up to 2 years. Pirfenidoneassociated adverse drug reactions (ADR) were collected.

Results At baseline, mean \pm SD age was $70 \pm 8.5$ years and mean \pm SD time since IPF diagnosis was $1.6 \pm 2.5$ years; $80 \%$ of patients were male; supplemental oxygen was used by $27 \%$ of patients; mean \pm SD FVC was $2.56 \pm 0.78 \mathrm{~L}$; mean \pm SD $\%$ predicted FVC was $66 \pm 16 \%$ (14\% had $<50 \%$ predicted FVC). The most common comorbidities (>10\%) were hypertension, gastroesophageal reflux disease, hypercholesterolemia and coronary artery disease.

At this interim analysis, median time on pirfenidone was 7.6 months and total exposure was 803 patient-years. Overall, 67\% of patients had $\geq 1 \mathrm{ADR}$, most commonly: nausea, $17 \%$; fatigue, $15 \%$; decreased appetite, $13 \%$; decreased weight, $12 \%$; rash, 10\%; and diarrhoea, 9\%. Of patients who had an ADR, 55\% experienced their first ADR within the first 30 days' treatment. Around $5 \%$ of patients completed 2 years' treatment, $55 \%$ are ongoing, 9\% died and 21\% discontinued due to pirfenidonerelated ADRs (most commonly nausea, rash and decreased weight). $11 \%$ discontinued for other reasons.

Patients with FVC $<50 \%$ had a higher discontinuation rate than other patients ( $48 \%$ vs $39 \%$, respectively). The imbalance was mainly driven by higher rates of death and lung transplantation. The discontinuation rate due to pirfenidone ADRs was similar among patients with FVC $<50 \%$ and $\geq 50 \%$ (20.3\% vs $20.9 \%$, respectively).

$62 \%$ of patients received pirfenidone alone; $11 \%, 8 \%$ and $8 \%$ received pirfenidone plus NAC, CS, or NAC+CS, respectively. The remaining $11 \%$ had partial use of NAC and/or steroids. ADR incidence was generally consistent for these subgroups except weight decrease and ALT increase, which occurred more often in the pirfenidone + CS group.

Conclusions In this real-world setting, pirfenidone was generally safe and well tolerated as monotherapy or combined with NAC and/or CS. The rate of discontinuation due to pirfenidonerelated ADRs was similar regardless of disease severity.

\section{P13 SAFETY OF PIRFENIDONE IN PATIENTS WITH IDIOPATHIC PULMONARY FIBROSIS (IPF): INTEGRATED ANALYSIS OF CUMULATIVE DATA FROM 5 CLINICAL TRIALS}

${ }^{1}$ PW Noble, ${ }^{2} \mathrm{C}$ Albera, ${ }^{3}$ WZ Bradford, ${ }^{4} \mathrm{U}$ Costabel, ${ }^{5}$ G Glaspole, ${ }^{6} \mathrm{MK}$ Glassberg, ${ }^{7} \mathrm{DJ}$ Lederer, ${ }^{3} \mathrm{Z}$ Lin, ${ }^{8} \mathrm{SD}$ Nathan, ${ }^{9} \mathrm{CA}$ Pereira, ${ }^{10} \mathrm{JJ}$ Swigris, ${ }^{11} \mathrm{D}$ Valeyre, ${ }^{12} \mathrm{~L}$ Lancaster. ${ }^{1} \mathrm{C}$ edars Sinai Medical Center, Los Angeles, USA; ${ }^{2}$ University of Turin, Turin, Italy; ${ }^{3}$ InterMune, Inc. (A Wholly Owned Roche Subsidiary), Brisbane, USA; ${ }^{4}$ Ruhrlandklinik, Essen, Germany; ${ }^{5}$ Alfred Hospital, Melbourne, Australia; ${ }^{6}$ University of Miami Miller School of Medicine, Miami, USA; ${ }^{7}$ Columbia University, New York, USA; ${ }^{8}$ Inova Fairfax Hospital, Falls Church, USA; ${ }^{9}$ Paulista School of Medicine, Federal University, Sao Paulo, Brazil; ${ }^{10}$ National Jewish Health, Denver, USA; "11Avicenne Univsersity Hospital, Bobigny, France; ${ }^{12}$ Vanderbilt University Medical Center, Nashville, USA

\subsection{6/thoraxjnl-2015-207770.150}

Introduction and objectives IPF is a chronic, progressive and irreversible disease that requires long-term clinical management. To further evaluate the clinical safety of pirfenidone in patients with IPF, we performed a comprehensive integrated analysis of safety data from 5 clinical trials.
Methods All patients assigned to receive pirfenidone $(2403 \mathrm{mg} /$ d) in the Phase 3 ASCEND (016) and CAPACITY (004/006) studies and all patients receiving $\geq 1$ dose of pirfenidone in either of two ongoing open-label studies (studies 002 and 012) comprised the integrated population. EAP (002) is a compassionate use study in the U.S.; RECAP (012) is evaluating pirfenidone in patients who completed one of the Phase 3 studies. Analyses were based on the January 15, 2014 interim data cut.

Results 1299 patients were included in the integrated population. The cumulative total exposure to pirfenidone was 3160 person exposure years (PEY). The median duration of exposure was 1.7 years (range, 1 week-9.9 years); 545 (42\%) patients received pirfenidone for $\geq 2$ years and 325 (25\%) patients received pirfenidone for $\geq 4$ years. The majority of patients $(75.8 \%)$ received a mean daily dose of $\geq 1800 \mathrm{mg}$. Consistent with prior observations, gastrointestinal and skin-related events were among the most common treatment emergent adverse events (Table 1); these were almost always mild to moderate in severity, reversible with dose modification and rarely led to treatment discontinuation. Cough, dyspnoea and IPF were the most common respiratory adverse events in the integrated population - a finding that is consistent with expectations in patients with a chronic progressive respiratory disease followed over a long period of observation. Aminotransferase (ALT or AST) elevations $(>3 \times$ ULN) occurred in 40/1299 (3.0\%) patients in the integrated population.

Abstract P13 Table 1 Treatment emergent adverse events in the integrated population compared with the pooled pirfenidone 2403 $\mathrm{mg} / \mathrm{d}$ and placebo groups in the Phase 3 trials*

\begin{tabular}{ll}
\hline & $\begin{array}{c}\text { Integrated population }(\mathrm{N}=1299)^{\dagger} \\
\text { OE = treatment emergent adverse event ment } \\
\text { emergent adverse events d sun exposure during } \\
\text { treatment with pirfenidone. the skin du }\end{array}$ \\
\hline Median (range) duration of exposure, yr & $1.7(>0,9.9)$ \\
Treatment emergent adverse event, \% & \\
Nausea & 37.6 \\
Cough & 35.1 \\
Dyspnea & 30.9 \\
Upper respiratory tract infection & 30.6 \\
Idiopathic pulmonary fibrosis & 29.3 \\
Fatigue & 28.2 \\
Diarrhoea & 28.1 \\
Rash & 25.0 \\
Bronchitis & 23.8 \\
Headache & 21.6 \\
Nasopharyngitis & 21.3 \\
Dizziness & 21.2 \\
Dyspepsia & 18.4 \\
Vomiting & 15.9 \\
Weight decreased & 15.6 \\
Back pain & 15.4 \\
Anorexia & 15.2 \\
\hline
\end{tabular}

${ }^{*}$ Occurring in $\geq 15 \%$ of patients in the cumulative clinical database.

"Includes 2 patients in Study 002 with a diagnosis of "pulmonary fibrosis." 
Conclusions A comprehensive integrated analysis of safety outcomes in a large, well-defined cohort of 1299 patients with IPF who were treated with pirfenidone for up to 9.9 years demonstrated that treatment with pirfenidone is safe and generally well tolerated. These observations provide further evidence to support the long-term clinical safety of pirfenidone in patients with IPF.

\section{P14 PIRFENIDONE IS EFFICACIOUS IN PATIENTS WITH IDIOPATHIC PULMONARY FIBROSIS (IPF) WITH MORE PRESERVED LUNG FUNCTION}

${ }^{1} \mathrm{PW}$ Noble, ${ }^{2} \mathrm{WZ}$ Bradford, ${ }^{3} \mathrm{U}$ Costabel, ${ }^{4}$ G Glaspole, ${ }^{5} \mathrm{MK}$ Glassberg, ${ }^{2} \mathrm{E}$ Gorina, ${ }^{2} \mathrm{D}$ Kardatzke, ${ }^{6} \mathrm{~L}$ Lancaster, ${ }^{7} \mathrm{DJ}$ Lederer, ${ }^{8} \mathrm{SD}$ Nathan, ${ }^{9} \mathrm{C}$ Pereira, ${ }^{10} \mathrm{D}$ Spirig, ${ }^{11} \mathrm{JJ}$ Swigris, ${ }^{12} \mathrm{D}$ Valeyre, ${ }^{13} \mathrm{C}$ Albera. ${ }^{1} \mathrm{C}$ dedars-Sinai Medical Center, Los Angeles, California, USA; IInterMune, Inc. (A Wholly Owned Roche Subsidiary), Brisbane, California, USA; ${ }^{3}$ Ruhrlandklinik and University of Duisburg - Essen, Essen, Germany; ${ }^{4}$ Alfred Hospital, Melbourne, Australia; ${ }^{5}$ University of Miami Miller School of Medicine, Miami, Florida, USA; ${ }^{6}$ Vanderbilt University Medical Center, Nashville, Tennessee, USA; ${ }^{7}$ Columbia University Medical Center, New York, New York, USA; ${ }^{8}$ Inova Fairfax Hospital, Falls Church, Virginia, USA; ${ }^{9}$ Paulista School of Medicine, Federal University of São Paulo, São Paulo, Brazil; ${ }^{10} \mathrm{~F}$. Hoffman-La Roche Ltd, Basel, Switzerland; " National Jewish Health, Denver, Colorado, USA; ${ }^{12}$ Assistance Publique-Hôpitaux de Paris, Hôpital Avicenne, Bobigny, France; ${ }^{13}$ University of Turin, Turin, Italy

\subsection{6/thoraxjnl-2015-207770.151}

Introduction and objectives IPF is a progressive, irreversible and fatal disease. Early treatment initiation when lung function is relatively preserved may have beneficial outcomes; however, published data to support this hypothesis are lacking. We investigated the efficacy of pirfenidone at 12 months in patients stratified by lung function using forced vital capacity (FVC) or GAP stage.

Methods Efficacy outcomes (FVC, 6-minute walk distance [6MWD] and dyspnea [UCSD SOBQ]) were analysed at 12 months in patients randomised to pirfenidone $2403 \mathrm{mg} / \mathrm{d}$ or placebo in the pooled CAPACITY/ASCEND population $(\mathrm{N}=$ 1247), stratified by baseline FVC $(\geq 80 \%,<80 \%)$ and GAP stage (GAP I, GAP II-III). Treatment-by-subgroup interaction was tested based on a rank ANCOVA model. The factors in the model included study, region, treatment, subgroup and treatment-by-subgroup interaction term.

Results Demographic characteristics were similar across all four groups. In the placebo arm, disease progression as measured by FVC occurred with comparable frequency in patients with FVC $\geq 80 \%$ and FVC $<80 \%$, as well as in patients with GAP I and GAP II-III stage. A higher proportion of placebo patients with FVC $<80 \%$ and GAP II-III stage had a $\geq 50-m$ decline in $6 \mathrm{MWD}$ or death or a $\geq 20$-point change in the UCSD SOBQ total score. Pirfenidone treatment reduced the proportion of patients experiencing a $\geq 10 \%$ FVC decline or death and increased the proportion of patients with no FVC decline in all subgroups. Pirfenidone also reduced the proportion of patients with $\geq 50-\mathrm{m}$ decline in the $6 \mathrm{MWD}$ or death and increased the proportion of patients with no 6MWD decline in all subgroups. The magnitude of treatment effect in patients with less $v s$ more preserved lung function was comparable, with no significant treatment-by subgroup interaction (Figure 1).

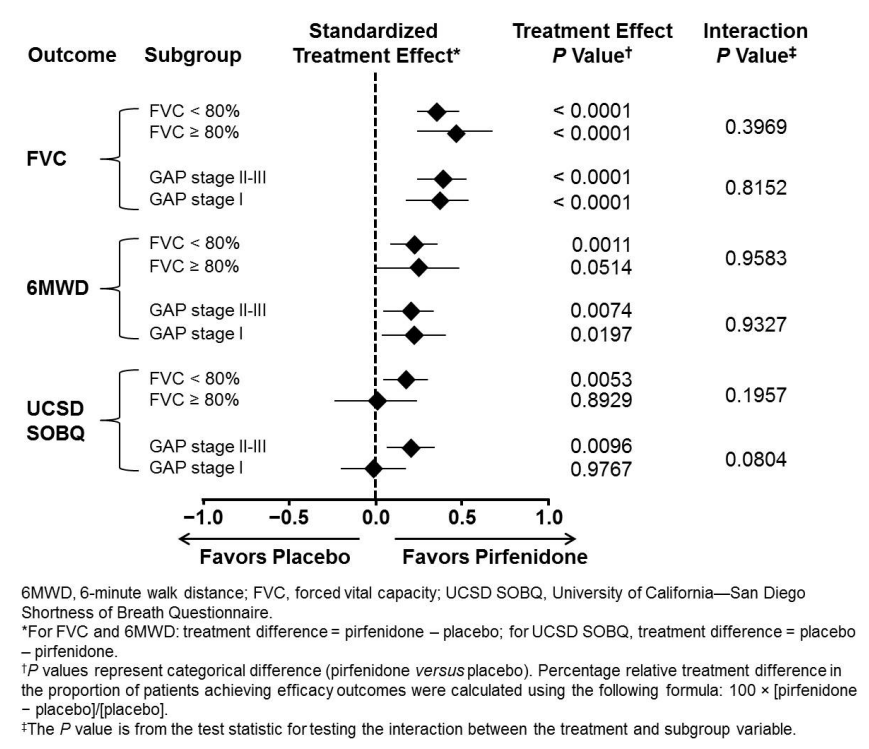

Abstract P14 Figure 1 Treatment effect of pirfenidone by baseline lung function

Conclusions In the placebo population, clinically significant disease progression occurs in subgroups with more and less preserved lung function at baseline, underlying the need for early intervention. The magnitude of pirfenidone treatment effect on functional measures was comparable in these subgroups of patients (FVC $<80 \%$ vs FVC $\geq 80 \%$ or GAP I vs GAP II-III stage), supporting the initiation of treatment soon after diagnosis, when pulmonary function is relatively preserved.

\section{Clinical studies of advanced COPD}

\section{P15 REGIONAL CEREBRAL ATROPHY AND COGNITIVE FUNCTION IN CHRONIC OBSTRUCTIVE PULMONARY DISEASE}

${ }^{1} \mathrm{CC}$ Savage, ${ }^{1} \mathrm{C}$ Pennington, ${ }^{2} \mathrm{PW}$ Jones, ${ }^{1} \mathrm{JW}$ Dodd. ${ }^{1}$ University of Bristol, Bristol, UK; ${ }^{2} \mathrm{St}$ George's University of London, London, UK

\subsection{6/thoraxjnl-2015-207770.152}

Background Widespread white matter damage and cognitive impairment have been demonstrated in COPD. However, it remains unclear if regional atrophy is present. We used a simple clinical visual rating scale to measure regional atrophy in a wellcharacterised population with COPD and compared age-matched controls. We explored correlations with demographics, disease factors and cognitive measures.

Objectives

1. a) To determine if there are significant differences in regional atrophy between COPD patients and age-matched control subjects.

2. b) To investigate whether patient characteristics or measures of disease severity account for group differences in atrophy severity.

3. c) To seek correlations with regional atrophy.

Methods A validated visual analogue MRI grading technique was used to assess the degree of atrophy in multiple brain regions in stable non-hypoxaemic COPD patients $(\mathrm{n}=25)$ and age- 